\title{
Axion-driven hybrid inflation over a barrier
}

\author{
Jinn-Ouk Gong ${ }^{1,2}$ and Kwang Sik Jeong $\oplus^{3}$ \\ ${ }^{1}$ Department of Science Education, Ewha Womans University, Seoul 03760, Korea \\ ${ }^{2}$ Asia Pacific Center for Theoretical Physics, Pohang 37673, Korea \\ ${ }^{3}$ Department of Physics, Pusan National University, Busan 46241, Korea
}

(Received 29 January 2021; accepted 23 July 2021; published 9 August 2021)

\begin{abstract}
We present a novel cosmological scenario that describes both inflation and dark matter. A concrete realization of our scenario is given based on a well-established particle physics model, where an axionlike field drives inflation until a potential barrier, which keeps a waterfall field at the origin, disappears to trigger a waterfall transition. Such a barrier makes the inflaton potential much flatter, improving significantly the naturalness and viability of the otherwise problematic setup adopted previously. The observed spectrum of the cosmic microwave background indicates that the inflationary Hubble scale, which is allowed to span a wide range, uniquely fixes the inflaton mass and decay constant. This raises an intriguing possibility of probing inflation via experimental searches for axionlike particles. Further, our model involves dark matter candidates including the inflaton itself. Also, for a complex waterfall field, we can determine cosmologically the Peccei-Quinn scale associated with the strong $C P$ problem.
\end{abstract}

DOI: 10.1103/PhysRevD.104.043511

\section{INTRODUCTION}

Cosmic inflation [1-3] has become an essential part of the standard cosmological model. Before the onset of the hot big bang evolution, it provides the necessary initial conditions-otherwise extremely finely tuned-as confirmed by the observations on the cosmic microwave background (CMB) [4]. Furthermore, it explains the origin of temperature fluctuations of the CMB and the inhomogeneous distribution of galaxies on large scales due to quantum fluctuations during inflation [5]. The properties of these primordial perturbations have been constrained by decades of observations, and are consistent with the predictions of inflation [6].

To implement inflation, we need typically an inflaton field with a sufficiently flat potential. The inflaton drives an inflationary epoch until the "slow-roll" period does not hold any longer [7,8]. It is, however, a formidable task to maintain an unusually flat potential against various corrections [9]. A powerful way of protecting the flatness is to impose certain symmetries. An axionlike field is an appealing candidate for an inflaton because its mass requires breaking of the associated shift symmetry, naturally making it very light. However, the predictions of the minimal axion-driven inflation — natural inflation [10,11]—are only

Published by the American Physical Society under the terms of the Creative Commons Attribution 4.0 International license. Further distribution of this work must maintain attribution to the author(s) and the published article's title, journal citation, and DOI. Funded by SCOAP . marginally consistent with the most recent Planck observations. Additionally, for successful inflation, the axion should have a trans-Planckian decay constant $f \gtrsim 5 m_{\mathrm{Pl}}$ with $m_{\mathrm{Pl}} \equiv(8 \pi G)^{-1 / 2}[6]$, which may be outside the range of validity of an effective field theory description.

In this article, we present a novel cosmological scenario in which an axionlike field can drive inflation successfully and at the same time contribute to dark matter. The end of the inflationary phase is triggered by a waterfall transition like hybrid inflation [12]. The distinctive features of our model are two fold. First, the waterfall field $\chi$ is trapped at the origin during inflation by a potential barrier. This implies that, differently from the previous hybrid inflation models, the scale of inflation is not tied to that of the waterfall phase transition. As a result, unlike the original natural inflation, the inflaton is allowed to have a decay constant well below $m_{\mathrm{Pl}}$ so that the effective field theory is trustable, yet maintains a flat potential. Thus the naturalness and viability of the models, which were problematic either in the effective theory viewpoint or in unnaturally fine-tuned initial conditions, are improved significantly within our scenario. The Planck results are accommodated in a broad range of the inflaton mass and decay constant, but with a certain relationship between them. This opens an interesting possibility to probe inflation via experimental searches for axionlike particles.

Another merit of our scenario is that the inflaton itself can constitute dark matter, which is generally difficult in other inflation models. Further, because the inflationary Hubble scale $H_{\text {inf }}$ is allowed to span a very wide range, $\chi$ has a potential to resolve other puzzles of the Standard 
Model (SM). If complex, we may identify $\mathrm{U}(1)_{\chi}$ with the Peccei-Quinn (PQ) symmetry solving the strong $C P$ problem [13]. Remarkably, the PQ scale is then determined cosmologically, and the contribution of the QCD axion to dark matter constrains $H_{\text {inf }}$ to be below about $10^{4} \mathrm{GeV}$.

\section{MODEL}

Our model consists of two scalar fields, the inflaton $\phi$ and the waterfall field $\chi$. During inflation, $\phi$ rolls down the potential slowly while $\chi$ is trapped at the origin by a barrier. There, the effective mass squared of $\chi, \mu_{\text {eff }}^{2}$, is thus positive. As $\phi$ evolves, $\mu_{\text {eff }}^{2}$ decreases monotonically and vanishes at a critical value $\phi_{c}$, removing the barrier. Then inflation ends almost instantaneously. Here, the barrier does bring the separation between the scales for inflation and waterfall phase transition.

Our scenario is successfully realized if $\phi$ is an axionlike field with decay constant $f$. This is because its interactions are well controlled by shift symmetry, $\phi \rightarrow \phi+$ constant, presumably broken only by nonperturbative effects, and the size of its potential terms is finite and insensitive to $f$. A dangerous waterfall tadpole can be avoided by imposing a symmetry, for instance, $\mathrm{U}(1)_{\chi}$ if $\chi$ is a complex scalar. Explicitly, we consider the potential

$V(\phi, \chi)=V_{0}+\mu_{\mathrm{eff}}^{2}(\phi)|\chi|^{2}-\lambda|\chi|^{4}+\frac{1}{\Lambda^{2}}|\chi|^{6}+U(\phi)$,

with $\lambda>0$, and the $\phi$-dependent terms given respectively by

$$
\begin{gathered}
\mu_{\mathrm{eff}}^{2}(\phi)=m^{2}-\mu^{2} \cos \left(\frac{\phi}{f}+\alpha\right), \\
U(\phi)=M^{4} \cos \left(\frac{\phi}{f}\right),
\end{gathered}
$$

where $\alpha$ is constant. The positive constant $V_{0}$ is fixed by demanding $V=0$ at the true vacuum, and $\Lambda$ is the cutoff scale of the theory. The parameter space of our interest is

$$
m^{4}<\mu^{4} \ll \lambda V_{0} \quad \text { and } \quad M^{4} \ll V_{0} .
$$

Then the true vacuum appears at $\chi_{0} \sim \sqrt{\lambda} \Lambda$, well below the cutoff scale $\Lambda$ as long as $\lambda \ll 1$, and $V_{0}$ reads

$$
V_{0} \sim \lambda^{3} \Lambda^{4}
$$

Note that there are two minima along the $\chi$-direction for $\mu_{\text {eff }}^{2}(\phi)>0$, and a barrier separates them. The position and height of the waterfall barrier are determined by $\mu$, whereas the value of $V_{0}$ is insensitive to it.

Before going further, let us discuss the case $\lambda<0$ so that there is no barrier, similar to hybrid natural inflation
[14-16]. In such a case, $V_{0}$ is fixed by $\mu$ roughly to be $\mu^{4} /|\lambda|$, and the possible range of $M^{4} / V_{0}$ is severely constrained because a closed loop of $\chi$ generally makes $|\lambda| \gtrsim 1 / 16 \pi^{2}$ and $M^{4} \gtrsim \mu^{2} \Lambda_{*}^{2} / 16 \pi^{2}$ with $\mu<\Lambda_{*}$. Here $\Lambda_{*}$ is the cutoff scale of the $\phi$-dependent waterfall mass operator. In our scenario, $M^{4} / V_{0}$ can be arbitrarily small, making the inflaton potential much flatter than the case without a barrier.

The rate of tunneling over a barrier is proportional to $\exp \left(-S_{E}\right)$, where $S_{E}$ is the Euclidean action of $\chi$ evaluated on a bounce solution. Tunneling proceeds dominantly via the Coleman-De Luccia bounce [17] with $S_{E}>S_{0} \equiv$ $8 \pi^{2} /(3 \lambda)$ in the region with $\mu_{\text {eff }}^{2}>2 H_{\text {inf }}^{2}$, while through the Hawking-Moss instantons [18] with $S_{E}=\mu_{\text {eff }}^{4} / H_{\text {inf }}^{4} \times S_{0}$ in the opposite region [19]. Here we have used that the bounce is insensitive to the $|\chi|^{6}$ term for $\mu^{4} \ll \lambda V_{0}$. For viable inflation, we thus impose the condition

$$
\mu^{2} \gg H_{\mathrm{inf}}^{2} .
$$

Then $\chi$ is heavy enough to be initially fixed at the origin. In addition, the tunneling rate is exponentially suppressed so that $\chi$ stays at the origin until the barrier disappears at $\phi_{c}$. Bubbles of true vacuum can be nucleated around the end of inflation, but the $\mathrm{U}(1)_{\chi}$ phase transition occurs rather smoothly because the barrier soon disappears.

As a simple ultraviolet completion of the inflaton potential, we consider a hidden $\mathrm{QCD}$ with $\mathrm{U}(1)_{\chi}$ charged quarks. (2) and (3) are then generated in a controllable way while naturally satisfying the hierarchies (4). Vectorlike quarks $u+u^{c}$ and $d+d^{c}$ couple to $\chi$ through the $\mathrm{U}(1)_{\chi}$ and gauge invariant interactions

$m_{u} u u^{c}+y \chi u^{c} d+y^{\prime} \chi^{*} u d^{c}+m_{d} d d^{c}+\frac{1}{16 \pi^{2}} \frac{\phi}{f} G_{\mu \nu} \tilde{G}^{\mu \nu}$,

where the hidden confining scale lies in the range $m_{d} \ll \Lambda_{h} \ll m_{u}$. Here we have taken the field basis where the quark mass parameters are real. Note that the last term above is an anomalous inflaton coupling to hidden gluons, which is the only source of shift symmetry breaking. At energy scales below $m_{u}, u+u^{c}$ are integrated out to give a $\chi$-dependent effective quark mass

$$
\left(\frac{y y^{\prime}}{m_{u}}|\chi|^{2}+m_{d}+\delta m_{d}\right) d d^{c}
$$

Here we have included the radiative contribution from a closed loop of $\chi$

$$
\delta m_{d}=\frac{y y^{\prime}}{16 \pi^{2}} m_{u} \log \left(\frac{\Lambda^{2}}{m_{\chi}^{2}}\right),
$$


with $m_{\chi}$ being the mass of the radial component of $\chi$. For small values of $\chi, d+d^{c}$ are lighter than $\Lambda_{h}$ and condensate to form a meson with mass and decay constant around $\Lambda_{h}$. The inflaton mixes with the meson in the presence of anomalous coupling to hidden gluons, and finally the effective potential at scales below $\Lambda_{h}$ is obtained by integrating out the heavy meson

$$
\begin{aligned}
\Delta V_{\text {eff }}= & -\left|\frac{y y^{\prime}}{m_{u}}\right| \Lambda_{h}^{3} \cos \left(\frac{\phi}{f}+\beta_{1}\right)|\chi|^{2} \\
& +\left|m_{d}+\delta m_{d}\right| \Lambda_{h}^{3} \cos \left(\frac{\phi}{f}+\beta_{2}\right),
\end{aligned}
$$

where the constant phases are given by $\beta_{1}=\arg \left(y y^{\prime} / m_{u}\right)$ and $\beta_{2}=\arg \left(m_{d}+\delta m_{d}\right)$. It is clear that the above reduces to (2) and (3) with $\alpha=\beta_{1}-\beta_{2}$. Also, the hierarchies $\mu^{4} \ll$ $\lambda V_{0}$ and $M^{4} \ll V_{0}$ are satisfied naturally if

$$
H_{\text {inf }} \lesssim \Lambda_{h} \ll \Lambda,
$$

where we have used that $H_{\text {inf }}$ should be lower than $\Lambda_{h}$ since otherwise instanton effects become very weak. On the other hand, $m$ should be smaller than $\mu$ because inflation ends when the barrier disappears. The smallness of $m$ may be accommodated in more speculative idea like supersymmetry or anthropic selection.

\section{COSMOLOGICAL DYNAMICS}

\section{A. Inflation}

The Universe undergoes an inflationary phase while $\chi$ is trapped at the origin. During this stage, $\phi$ evolves down the potential

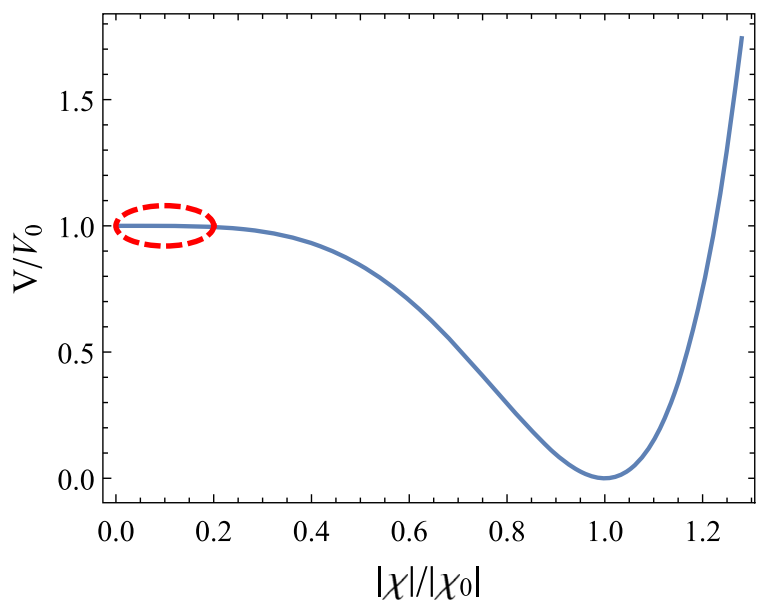

$$
V=V_{0}+U(\phi)=V_{0}+M^{4} \cos \left(\frac{\phi}{f}\right) .
$$

Thus, the evolution of $\phi$ during inflation is essentially identical to hybrid natural inflation. $\mu_{\text {eff }}^{2}$ crosses zero when $\phi$ reaches the critical value

$$
\frac{\phi_{c}}{f}=\cos ^{-1}\left(\frac{m^{2}}{\mu^{2}}\right)-\alpha .
$$

The sign flip triggers the waterfall phase transition, because there is no potential barrier along the $\chi$-direction, and inflation ends almost instantaneously. Among the model parameters, $m, \mu$, and $\alpha$ affect inflation only through the above combination. Figure 1 shows schematically the inflationary and waterfall phases.

At this point, it is very important to note that two crucial ingredients are required to make our scenario distinctive. One is the shift symmetry of $\phi$, which naturally allows the hierarchies (4). The other is a $\phi$-dependent barrier between two extrema at and off the origin in the waterfall potential. The barrier makes the inflaton potential flatter, and consequently the value of $f$ required for viable inflation can naturally be much lower than $m_{\mathrm{Pl}}$.

For $M^{4} \ll V_{0}$, inflation is driven by $V_{0}$ so that

$$
H_{\mathrm{inf}}^{2} \approx \frac{V_{0}}{3 m_{\mathrm{Pl}}^{2}} .
$$

From (12), the slow-roll parameters are given by, with $\theta \equiv \phi / f$,

$$
\begin{gathered}
\epsilon \equiv \frac{m_{\mathrm{Pl}}^{2}}{2}\left(\frac{V^{\prime}}{V}\right)^{2} \approx \frac{1}{2}\left(\frac{m_{\mathrm{Pl}}}{f}\right)^{2}\left(\frac{M^{4}}{V_{0}}\right)^{2} \sin ^{2} \theta, \\
\eta \equiv m_{\mathrm{Pl}}^{2} \frac{V^{\prime \prime}}{V} \approx-\left(\frac{m_{\mathrm{Pl}}}{f}\right)^{2}\left(\frac{M^{4}}{V_{0}}\right) \cos \theta .
\end{gathered}
$$

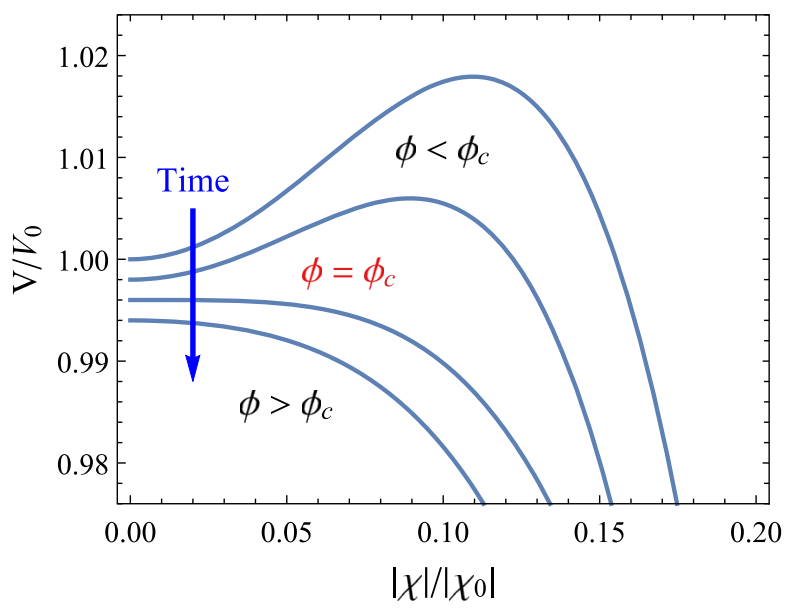

FIG. 1. Schematic display of inflationary and waterfall phases. The evolution of $\phi$ changes the waterfall potential dramatically near the origin, as illustrated in the right panel, but rarely around the true vacuum at $\chi=\chi_{0}$ if (4) holds. 
Thus, $|\eta|$ is parametrically much bigger than $\epsilon$. The slowroll conditions, $\epsilon \ll 1$ and $|\eta| \ll 1$, are satisfied if the following condition holds

$$
f \gtrsim\left(\frac{M^{4}}{V_{0}}\right)^{1 / 2} m_{\mathrm{Pl}}
$$

but it need not be above $m_{\mathrm{Pl}}$.

The amplitude of the power spectrum of the curvature perturbation and its spectral index, and the tensor-to-scalar ratio in terms of the slow-roll parameters are constrained as [6]

$$
\begin{gathered}
A_{\mathcal{R}}=\frac{V_{0}}{24 \pi^{2} m_{\mathrm{Pl}}^{4} \epsilon_{*}} \approx 2.0989_{-0.0292}^{+0.0296} \times 10^{-9}, \\
n_{\mathcal{R}}=1-6 \epsilon_{*}+2 \eta_{*} \approx 0.9656 \pm 0.0042, \\
r=16 \epsilon_{*}<0.056,
\end{gathered}
$$

where the subscript $*$ denotes the evaluation at the horizon exit. Since $\epsilon \ll|\eta|, n_{\mathcal{R}}$ is determined entirely by $\eta$. Hence, from (16) and (19), $f$ is written as

$$
\begin{aligned}
f & =\sqrt{\frac{2}{1-n_{\mathcal{R}}} \cos \theta_{*}}\left(\frac{M^{4}}{V_{0}}\right)^{1 / 2} m_{\mathrm{Pl}} \\
& \approx 7.625 \sqrt{\cos \theta_{*}}\left(\frac{M^{4}}{V_{0}}\right)^{1 / 2} m_{\mathrm{Pl}}
\end{aligned}
$$

while (20) is translated to the following mild constraint

$$
\frac{M^{4}}{V_{0}}<\frac{0.056}{8} \frac{2}{1-n_{\mathcal{R}}} \cos \theta_{*} \approx 0.4070 \cos \theta_{*} .
$$

The number of $e$-folds $N$, before the onset of the waterfall phase transition, is estimated by

$$
\begin{aligned}
N & =\frac{1}{m_{\mathrm{Pl}}} \int_{\phi_{c}}^{\phi} \frac{d \phi^{\prime}}{\sqrt{2 \epsilon}} \approx \frac{V_{0}}{M^{4}}\left(\frac{f}{m_{\mathrm{Pl}}}\right)^{2} \log \left[\frac{\tan \left(\theta_{c} / 2\right)}{\tan (\theta / 2)}\right] \\
& \approx 58.14 \cos \theta_{*} \log \left[\frac{\tan \left(\theta_{c} / 2\right)}{\tan (\theta / 2)}\right],
\end{aligned}
$$

thus we can use $\theta$ and $N$ interchangeably. The required number of $e$-folds is around 60, which fixes $\theta_{*}$ roughly as

$$
\theta_{*} \approx 0.7126 \tan \left(\frac{\theta_{c}}{2}\right)-0.1566 \tan ^{3}\left(\frac{\theta_{c}}{2}\right)
$$

neglecting terms of higher order in $\tan \left(\theta_{c} / 2\right)$. Thus, $\theta_{*}$ does not need to be very close to the hilltop of the potential.

The inflaton mass during inflation sets the lower bound of the inflaton mass at the true vacuum, $\left.m_{\phi}\right|_{\min }=M^{2} / f$. It is interesting to note that both $f$ and $\left.m_{\phi}\right|_{\min }$ are proportional

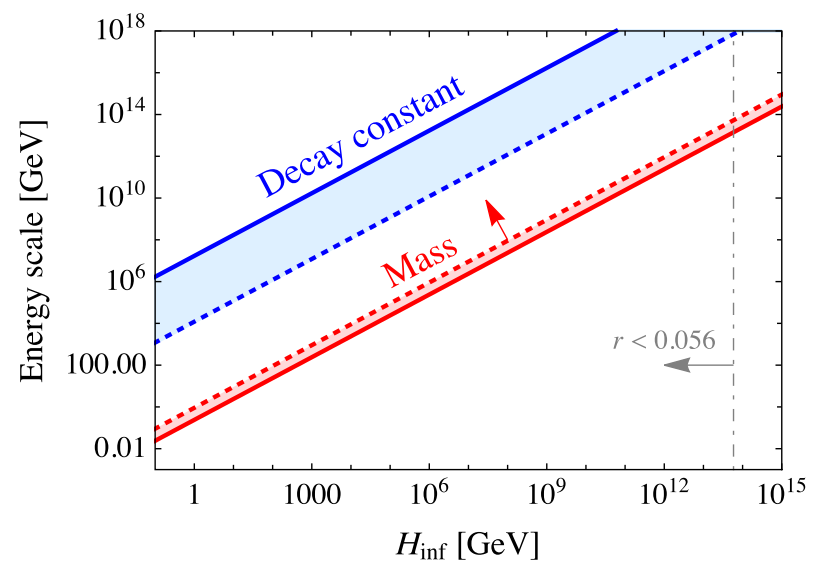

FIG. 2. Decay constant and lower bound on mass of the inflaton compatible with the Planck results on $n_{\mathcal{R}}$ and $A_{\mathcal{R}}$. We have taken $\theta_{*}$ lying between 0.01 (solid lines) and 1.5 (dotted lines).

to $H_{\text {inf }}$; combining (18) with (14) and (21), they are written respectively as

$$
\begin{gathered}
f=\frac{H_{\mathrm{inf}}}{\pi\left(1-n_{\mathcal{R}}\right) \sqrt{A_{\mathcal{R}}} \tan \theta_{*}} \approx \frac{2.020 \times 10^{5}}{\tan \theta_{*}} H_{\mathrm{inf}}, \\
\left.m_{\phi}\right|_{\min }=\sqrt{\frac{3\left(1-n_{\mathcal{R}}\right)}{2 \cos \theta_{*}}} H_{\mathrm{inf}} \approx \frac{0.2272}{\sqrt{\cos \theta_{*}}} H_{\mathrm{inf}} .
\end{gathered}
$$

Figure 2 shows the relationship between $H_{\text {inf }},\left.m_{\phi}\right|_{\min }$, and $f$. $\phi$ can couple to the SM sector, for instance, to gauge bosons through anomalous couplings as naturally expected from its axionic nature. Our scenario thus provides theoretical support for experimental searches for axionlike particles in a wide mass range. The rough relation, $f \sim 10^{6} \times\left. m_{\phi}\right|_{\min }$, indicates that $\phi$ should be heavier than about $0.1 \mathrm{GeV}$ to avoid too rapid cooling of stars [20], if coupled to photons. Another plausible possibility is that it instead couples to the Higgs sector as in the cosmological relaxation model of the weak scale [21]. Then, it may be detectable at collider and beam-dump experiments. For instance, the mass range below a few $\mathrm{GeV}$ can be probed by experiments at SHiP [22] and NA62 [23].

The postinflationary evolution leads to very rich phenomenologies. The quantitative predictions depend very much on the detail of the model, so here we are satisfied with describing briefly the subsequent evolution. After the barrier disappears, $\chi$ soon acquires a tachyonic mass much larger than $H_{\text {inf }}$ in magnitude for $\mu^{2} \gg H_{\text {inf. }}^{2}$ This happens within an $e$-fold after $\phi=\phi_{c}$, so $\chi$ rolls fast down to the true vacuum. Unlike usual axion-driven inflation where the Universe is reheated via an anomalous coupling to photons, the reheating process depends greatly on the details of the model. Generally speaking, however, depending on the couplings, tachyonic preheating [24] is extremely effective so that the vacuum energy is rapidly transferred to the 
energy of inhomogeneous oscillations of $\phi$ and/or $\chi$ $[25,26]$, subsequently heating up the Universe to a radiation-dominated regime.

After inflation, spontaneous $\mathrm{U}(1)_{\chi}$ breaking occurs and leads to the formation of cosmic strings [27], which can survive in the late Universe and contribute to the $\mathrm{CMB}$ temperature anisotropies, depending on how the associated Nambu-Goldstone boson becomes massive. For instance, for global $\mathrm{U}(1)_{\chi}$, it can obtain a mass nonperturbatively from some confining gauge sector. Then, topological defects are unstable and collapse by the wall tension if the domain-wall number is equal to unity, or if a small explicit symmetry-breaking term is added to lift the vacuum degeneracy [28,29]. Further, cosmic string loops and large time-dependent inhomogeneities generated during tachyonic preheating can act as a source of gravitational waves (GWs). The corresponding GW spectrum can span a huge range of frequencies; from $\mathcal{O}\left(10^{-12}\right) \mathrm{Hz}$ to $\mathcal{O}(1) \mathrm{Hz}$ for stable and metastable cosmic strings [30], within the reach of pulsar-timing arrays, LIGO, and LISA, and from $\mathcal{O}(1) \mathrm{Hz}$ to $\mathcal{O}\left(10^{10}\right) \mathrm{Hz}$ for inhomogeneities from tachyonic preheating [31-33]. Such high-frequency GWs are unfortunately beyond the sensitivity of interferometric experiments due to the short-noise fluctuations of photons. GWs in relatively low-frequency regimes may well be within the reach of future detectors like advanced LIGO, the Einstein Telescope, and the Big Bang Observer, which however is possible only for extremely small values of couplings.

\section{B. Dark matter}

Another distinctive feature of our scenario is the possibility that the inflaton can contribute to dark matter if its potential arises from hidden QCD as in (7). Having Yukawa interactions with $\chi$, the hidden quarks have masses increasing with the waterfall field value. This implies that $\Lambda_{h}$ also increases, and thus the hidden QCD gets stronger after inflation. In the region of large waterfall field values where all the hidden quarks are heavier than $\Lambda_{h}$, we have

$$
\mu^{2}=0 \quad \text { and } \quad M^{4}=\Lambda_{h}^{4},
$$

in (2) and (3), because there are no mesons formed.

Let us consider the case that $\chi_{0}$ is sufficiently large so that (27) holds in the present Universe. $\phi$ is then stabilized at a $C P$-conserving minimum, and consequently accidental $Z_{2}$ symmetry arises; $\phi \rightarrow-\phi$. The $Z_{2}$ forbids $\phi$ to mix with $\chi$, making it stable if does not couple to SM. $\phi$ starts coherent oscillations around the minimum when $H$ becomes comparable to its mass, i.e., at the temperature fixed by

$$
m_{\phi}\left(T_{1}\right)=3 H\left(T_{1}\right)
$$

If $T$ is below $\Lambda_{h}$ during reheating, oscillations start before reheating ends. Then, the inflaton relic density from oscillation is roughly estimated by

$\Omega_{\phi} h^{2} \sim 0.24 \theta_{c}^{2}\left(\frac{T_{1}}{\Lambda_{h}}\right)^{n}\left(\frac{f}{10^{11} \mathrm{GeV}}\right)^{2}\left(\frac{T_{\text {reh }}}{10^{5} \mathrm{GeV}}\right)$,

with $T_{1}<\Lambda_{h}$, and $n=11 N / 6-2$ for confining $\mathrm{SU}(N)$. Here $T_{\text {reh }}$ is the reheating temperature at which the Universe becomes completely radiation-dominated, and we have used that the scale factor scales as $\mathrm{H}^{-2 / 3}$ during a matter-dominated era. If oscillations start after reheating, the relic density can be read off from (29) by replacing $T_{\text {reh }}$ with $T_{1} . \phi$ can thus account for the observed dark matter in a wide range of $f$ depending on $T_{\text {reh. }}$. It is worth noting that $\alpha=0$ leads to accidental $Z_{2}$ even when (27) is not the case [34].

The inflation sector includes another candidate for dark matter associated with spontaneously broken $\mathrm{U}(1)_{\chi}$. An interesting possibility arising due to a wide allowed range of $H_{\text {inf }}$ is to identify $\mathrm{U}(1)_{\chi}$ with the PQ symmetry so that $\arg \chi$ becomes the QCD axion explaining the absence of $C P$ violation in $\mathrm{QCD}$. This implies that the waterfall and PQ phase transitions are identical. Then, as corresponds to $\chi_{0}$, the axion decay constant is cosmologically determined by

$$
f_{a} \approx \frac{3.8 \times 10^{11} \mathrm{GeV}}{\lambda^{1 / 4}}\left(\frac{H_{\mathrm{inf}}}{10^{4} \mathrm{GeV}}\right)^{1 / 2},
$$

which should be above about $10^{9} \mathrm{GeV}$ to avoid astrophysical bounds. The axion anomalous coupling to gluons, which is required to solve the strong $C P$ problem, is generated by adding $\mathrm{U}(1)_{\chi}$-charged heavy quarks or extra Higgs doublets $[35,36]$. We also note that the domain-wall number should be equal to one since otherwise domain walls formed during the QCD phase transition overclose the Universe. In such a case, axions are produced from coherent oscillations and, more efficiently, from unstable domain walls bounded by an axion string. The relic density is estimated by [37]

$$
\Omega_{a} h^{2} \approx 0.54 \times\left(\frac{\Lambda_{\mathrm{QCD}}}{400 \mathrm{MeV}}\right)\left(\frac{f_{a}}{10^{11} \mathrm{GeV}}\right)^{1.19} .
$$

Therefore, the observed dark-matter density indicates

$$
H_{\text {inf }} \lesssim \sqrt{\lambda} \times 10^{4} \mathrm{GeV}
$$

It would be also interesting to consider other cases where $\mathrm{U}(1)_{\chi}$ is identified, for instance, with $\mathrm{U}(1)_{L}$ associated with the seesaw mechanism or local $\mathrm{U}(1)_{B-L}$ to extend SM. 


\section{CONCLUSIONS}

We have proposed a cosmological scenario that improves significantly the naturalness and viability of axion-driven inflation. During inflation, the waterfall field remains at the origin by a potential barrier, which disappears when the inflaton reaches at a critical point - then inflation ends almost instantaneously. The inflaton interaction responsible for such a barrier can naturally arise if the shift symmetry is broken nonperturbatively by hidden QCD with quarks coupled to the waterfall field. Interestingly, the Planck results indicate the possibility of probing our scenario by experimental searches for axionlike particles. It is also remarkable that the inflaton can be stable enough to constitute dark matter if all the hidden quarks get heavier than the confining scale at the true vacuum. Further, for the case of a complex waterfall field, its phase component can play the role of the QCD axion, contributing to dark matter for $H_{\text {inf }}$ below about $10^{4} \mathrm{GeV}$.

\section{ACKNOWLEDGMENTS}

This work is supported in part by the National Research Foundation of Korea Grants No. 2018R1C1B6006061, No. 2021R1A4A5031460 (K. S. J.) and No. 2019R1A2C2085023 (J. G.). We also acknowledge the Korea-Japan Basic Scientific Cooperation Program supported by the National Research Foundation of Korea and the Japan Society for the Promotion of Science (2020K2A9A2A08000097). J. G. is further supported in part by the Ewha Womans University Research Grant of 2020 (1-2020-1630-001-1). J. G. is grateful to the Asia Pacific Center for Theoretical Physics for hospitality while this work was under progress.
[1] A. H. Guth, Phys. Rev. D 23, 347 (1981).

[2] A. D. Linde, Phys. Lett. B 108, 389 (1982).

[3] A. Albrecht and P. J. Steinhardt, Phys. Rev. Lett. 48, 1220 (1982).

[4] N. Aghanim et al. (Planck Collaboration), Astron. Astrophys. 641, A6 (2020).

[5] V. F. Mukhanov and G. V. Chibisov, JETP Lett. 33, 532 (1981), http://jetpletters.ru/ps/1510/article_23079.shtml.

[6] Y. Akrami et al. (Planck Collaboration), Astron. Astrophys. 641, A10 (2020).

[7] V. Mukhanov, Physical Foundations of Cosmology (Cambridge University Press, Cambridge, England, 2005), p. 421.

[8] S. Weinberg, Cosmology (Cambridge University Press, Cambridge, England, 2008), p. 593.

[9] D. H. Lyth and A. Riotto, Phys. Rep. 314, 1 (1999).

[10] K. Freese, J. A. Frieman, and A. V. Olinto, Phys. Rev. Lett. 65, 3233 (1990).

[11] F. C. Adams, J. R. Bond, K. Freese, J. A. Frieman, and A. V. Olinto, Phys. Rev. D 47, 426 (1993).

[12] A. D. Linde, Phys. Rev. D 49, 748 (1994).

[13] R. D. Peccei and H. R. Quinn, Phys. Rev. Lett. 38, 1440 (1977).

[14] G. G. Ross and G. German, Phys. Lett. B 684, 199 (2010).

[15] G. G. Ross and G. German, Phys. Lett. B 691, 117 (2010).

[16] G. G. Ross, G. German, and J. A. Vazquez, J. High Energy Phys. 05 (2016) 010.

[17] S. R. Coleman and F. De Luccia, Phys. Rev. D 21, 3305 (1980).

[18] S. W. Hawking and I. G. Moss, Phys. Lett. B 110, 35 (1982).

[19] A. Shkerin and S. Sibiryakov, Phys. Lett. B 746, 257 (2015).

[20] G. G. Raffelt, Annu. Rev. Nucl. Part. Sci. 49, 163 (1999).
[21] P. W. Graham, D. E. Kaplan, and S. Rajendran, Phys. Rev. Lett. 115, 221801 (2015).

[22] S. Alekhin et al., Rep. Prog. Phys. 79, 124201 (2016).

[23] S. Martellotti, arXiv:1510.00172.

[24] G. N. Felder, J. Garcia-Bellido, P. B. Greene, L. Kofman, A. D. Linde, and I. Tkachev, Phys. Rev. Lett. 87, 011601 (2001).

[25] J. Garcia-Bellido and A. D. Linde, Phys. Rev. D 57, 6075 (1998).

[26] E. J. Copeland, S. Pascoli, and A. Rajantie, Phys. Rev. D 65 , 103517 (2002).

[27] R. Jeannerot, J. Rocher, and M. Sakellariadou, Phys. Rev. D 68, 103514 (2003).

[28] G. B. Gelmini, M. Gleiser, and E. W. Kolb, Phys. Rev. D 39, 1558 (1989).

[29] S. E. Larsson, S. Sarkar, and P. L. White, Phys. Rev. D 55, 5129 (1997).

[30] P. Auclair, J. J. Blanco-Pillado, D. G. Figueroa, A. C. Jenkins, M. Lewicki, M. Sakellariadou, S. Sanidas, L. Sousa, D. A. Steer, J. M. Wachter et al., J. Cosmol. Astropart. Phys. 04 (2020) 034.

[31] J. Garcia-Bellido and D. G. Figueroa, Phys. Rev. Lett. 98, 061302 (2007).

[32] J. Garcia-Bellido, D. G. Figueroa, and A. Sastre, Phys. Rev. D 77, 043517 (2008).

[33] J.F. Dufaux, G. Felder, L. Kofman, and O. Navros, J. Cosmol. Astropart. Phys. 03 (2009) 001.

[34] S. H. Im and K. S. Jeong, Phys. Lett. B 799, 135044 (2019).

[35] A. Ringwald, Phys. Dark Universe 1, 116 (2012).

[36] M. Kawasaki and K. Nakayama, Annu. Rev. Nucl. Part. Sci. 63, 69 (2013).

[37] T. Hiramatsu, M. Kawasaki, K. Saikawa, and T. Sekiguchi, J. Cosmol. Astropart. Phys. 01 (2013) 001. 